\title{
Prolonged viability of human organotypic skin explant in culture method (hOSEC)
}

\author{
Marco Andrey Cipriani Frade ${ }^{1}$ \\ Andréia Fernanda Carvalho Leone Aguiar ${ }^{2}$ \\ Marcel Nani Leite ${ }^{1,2}$ \\ Eduardo Barbosa Coelho ${ }^{1}$
}

\author{
Thiago Antônio Moretti de Andrade ${ }^{2}$ \\ Flávia Araújo Guedes ${ }^{2}$ \\ Williane Rodrigues Passos ${ }^{2}$ \\ Pranab Kummar Das ${ }^{3,4,5,6}$
}

DOI: http:/ / dx.doi.org/10.1590/abd1806-4841.20153645

\begin{abstract}
A bstract: BACKGROUND: Currently, the cosmetic industry is overwhelmed in keeping up with the safety assessment of the increasing number of new products entering the market. To meet such demand, research centers have explored alternative methods to animal testing and also the large number of volunteers necessary for preclinical and clinical tests. OBJECTIVES: This work describes the human skin ex-vivo model (hOSEC: Human Organotypic Skin Explant Culture) as an alternative to test the effectiveness of cosmetics and demonstrate its viability through cutaneous keratinocytes' proliferative capacity up to 75 days in culture.

Methods: The skin explants obtained from surgeries were cultured in $\mathrm{CO}_{2}$-humid incubator. After 1, 7, 30 and 75 days in culture, skin fragments were harvested for analysis with histomorphological exam (HE staining) on all days of follow-up and immunohistochemistry for Ck5/6, Ck10 and Ki-67 only on the 75th day.

Results: On the 7th day, the epidermis was perfect in the dermoepidermal junction, showing the viability of the model. On the 30th day, the epidermis was thicker, with fewer layers on the stratum corneum, although the cutaneous structure was unaltered. On the 75th day, the skin became thinner but the dermoepidermal junctions were preserved and epidermal proliferation was maintained. After the 75th day on culture, the skin was similar to normal skin, expressing keratinocytes with Ck5/6 on supra-basal layers; Ck10 on differentiated layers; and viability could be assessed by the positivity of basal cells by Ki- 67 .

CONCLUSION: The hOSEC model seems a good alternative to animal testing; it can be used as a preclinical test analogous to clinical human skin test with similar effectiveness and viability proven by immunohistological analyses. Keywords: Animal use alternatives; Feasibility studies; Free tissue flaps; Innovation; Skin physiological processes; Tissue culture techniques
\end{abstract}

\section{INTRODUCTION}

Side effects caused by pharmaceutical and topical products are of increasing concern for patients, dermatologists and the chemical industry. Chemicals may be hazardous to the skin, inducing cutaneous irritation, burns and allergies. The regulatory guidelines, as proposed by the European Union, require that data on the level of irritancy of new chemicals must be reported. ${ }^{1}$ In addition, the irritancy potential of existing substances may need to be re-evaluated if current data are judged to be insufficient. Development of new compounds requires their safety assessment for humans and the environment.
A large number of animals, especially rabbits, are still used to test the dermal irritancy of chemical compounds. ${ }^{2}$ For ethical reasons, the use of experimental animals for skin irritation studies is not desirable, especially to test cosmetic products. A number of alternative methods to test for skin irritation have been proposed and several were evaluated at the ECVAM (European Centre for the Validation of Alternative Methods) in pre-validation trials, but none has been successfully pre-validated yet. ${ }^{3}$ Some of these methods are based on human keratinocyte culture to analyze the metabolism of dimethyl-thiazoldiphenyltetrazolium bromide (MTT) by cellular lactate dehydrogenase (LDH) con-

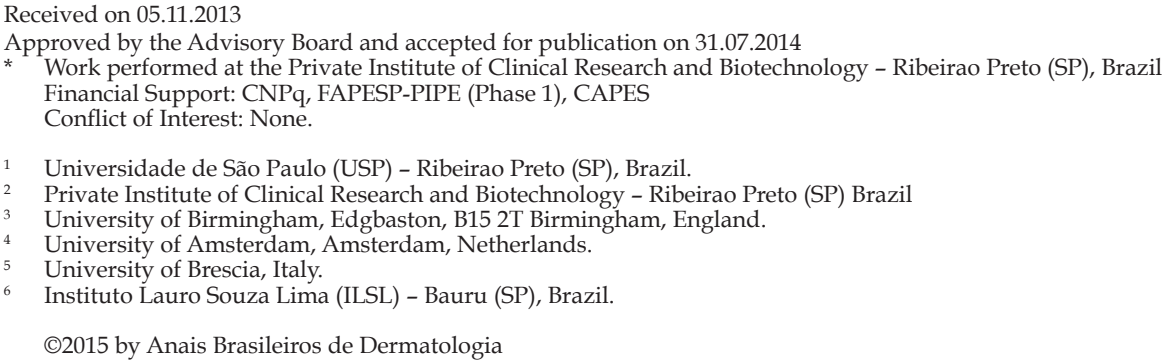


nections or neutral red from preloaded cells ${ }^{4-6}$ However, some disadvantages must be considered, since these analyses have to be made in a short timeframe and using only diluted products or chemicals in culture medium. Additionally, there is no skin resurfacing in these models to permit topical products to be directly applied, as occurs during in vivo testing.

The hOSEC (human organotypic skin explant culture) represents a model that is closer to in vivo human skin, like a 3D human skin model, consisting entirely of melanocytes, Langerhans cells, keratinocytes, and the whole structure of dermal fibroblasts, glycosaminoglycans and collagen, ideal for laboratory testing. Such features became essential for its differentiation from the existing three-dimensional validated models, which are composed only by keratinocytes and fibroblasts on a devitalized extracellular matrix. By presenting an air-liquid interface, the hOSEC system allows the application of creams, ointments and lipid-soluble substances on the horny layer, whereas in a cell culture system it is possible to add substances that are soluble in its medium due to the absence of a stratum corneum. . $^{7-10}$

Considering the complete structure of hOSEC as human skin, this model seems appropriate for testing of topical cutaneous products like sunscreen. Some authors have used the hOSEC model to analyze cell migration into the skin after chemical sensitization; others evaluate it through keratinocyte cytotoxicity by measuring the disappearance of keratinocytic RNA, which is visualized using a modified methyl green-pyronine (MGP) staining. ${ }^{7-10}$ Although these studies have demonstrated the viability of this model, in none of them was such viability longer than 3 weeks, and neither was the explanted skin's proliferative capacity.

So, the purpose of this study is to demonstrate the ex vivo model of human skin (hOSEC) as an alternative for sunscreen (sun protection factor - SPF) efficacy tests and demonstrate its viability through cutaneous keratinocytes' proliferative capacity for up to 75 days in culture.

\section{MATERIAL AND METHODS (hOSEC) \\ Human organotypic skin explant culture}

Skin was obtained with the informed consent of patients undergoing either breast or abdomen reduction surgery, in accordance with the ethical guidelines of the Research Ethics Committee of the Clinics Hospital at Ribeirao Preto Medical School, University of Sao Paulo (protocol number 1669/2014).

The explants were cut $\left(1.0 \mathrm{~cm}^{2}\right)$ and cultured on metal grids into standard 6-well plates in contact with culture medium at $37^{\circ} \mathrm{C}$ in $5 \% \mathrm{CO}_{2}$ humidified air (Figure 1). The culture media consist of Dulbecco's
Modified Eagle Medium (DMEM) with 10\% Fetal Bovine Serum (Sigma-Aldrich, Germany), 1\% anti-anti (10.000 units penicillin, $10 \mathrm{mg}$ streptomycin and $25 \mu \mathrm{g}$ amphotericin B - Sigma-Aldrich, Germany) and 1\% L-glutamine (Sigma-Aldrich, Germany). After 1, 7, 30 and 75 days in culture, the explants were harvested, fixed in formalin, embedded in paraffin in compliance with histology and immunohistochemistry methods. ${ }^{10}$

\section{Histology}

Paraffined sections $(3.0 \mu \mathrm{m})$ were stained with hematoxylin eosin (HE) for histomorphological analysis. Changes in epidermal morphology assessments were followed by qualitative analysis using LEICA $^{\circledR}$ DM-4000B optical microscope with a LEI$\mathrm{CA}^{\circledR}$ DFC 280 camera connected to the computer, with LAS $^{\circledast}$ - Leica Application for capturing images (Leica Microsystems,Germany) software.

\section{Immunohistochemistry}

Three-micrometer paraffined sections were subjected to antigen retrieval by autoclaving in citrate buffer, $\mathrm{pH}$ 6.0, for 5 minutes. Endogenous peroxidase was blocked with $3 \%$ hydrogen peroxide in PBS, followed by nonspecific blocking with $1 \%$ BSA. The sections were incubated with primary antibody in 1\% BSA overnight at $4^{\circ} \mathrm{C}$. The antibodies used were mouse anti-human Ck5/ 6 (1:100 dilution), Ck10 (1:100) and Ki67 (1:100) (Santa Cruz Biotechnology, USA). NovoLink Polymer Detection System Kit (Novocastra Laboratories Ltd, Newcastle Upon Tyne, UK) was used. The color reaction was developed by 3,3 diaminobenzidine tetrahydrochloride (Sigma-Aldrich, Germany). The sections were counterstained with Mayer's hematoxylin and mounted in Entelan (Sigma-Aldrich, Germany). The expression of Ck5/6 (basal cells of epidermis), Ck10 (stratum corneum) and Ki-67 (proliferative keratinocyte) were analyzed qualitatively only on the $75^{\text {th }}$ day of follow-up.



FIGURE 1: The explants were cut $\left(1.0 \mathrm{~cm}^{2}\right)$ and cultured on metal grids in standard 6-well plates, in contact with culture medium at $37^{\circ} \mathrm{C}$ in $5 \% \mathrm{CO}_{2}$ humidified air 


\section{RESULTS}

\section{Histological evaluation}

Histologically, the model showed viability on the $7^{\text {th }}$ day, as the appearance of the epidermis was similar to that of the $1^{\text {st }}$ day and it maintained a perfect connection on the dermoepidermal junction. Furthermore, on the $7^{\text {th }}$ day, the epidermis presented all the strata: basal, spinosum, granulosum and corneum. There were not any changes observed on the dermis. On the $30^{\text {th }}$ day the epidermis was thicker and with less stratum spinosum, although the cutaneous structure was unaltered and still viable to receive the ap- plication of topical products. On the $75^{\text {th }}$ day, the skin became thinner, but the dermoepidermal junction was unchanged and there was proliferation of the epidermis (Figure 2).

\section{Immunohistochemical evaluation}

After the $75^{\text {th }}$ day on culture, the skin at immunohistochemical examination was similar to normal skin, expressing keratinocytes with $\mathrm{Ck} 5 / 6$ on supra-basal layers; Ck10 on upper differentiated layers and presenting viability which could be confirmed by the positivity of basal cells to Ki-67. (Figure 3).

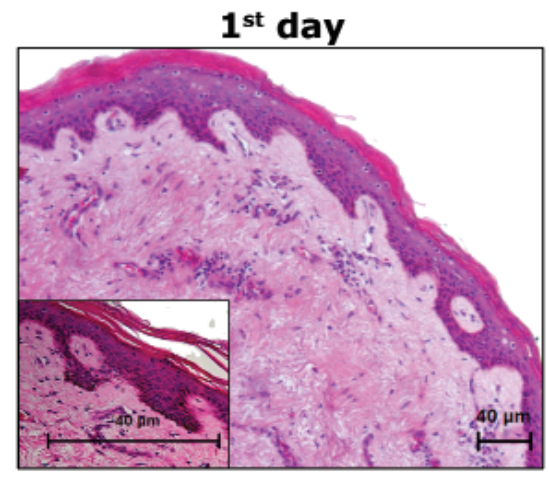

$30^{\text {th }}$ day

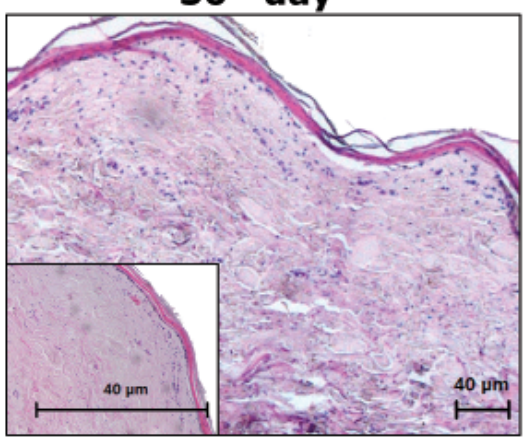

Ck5/6

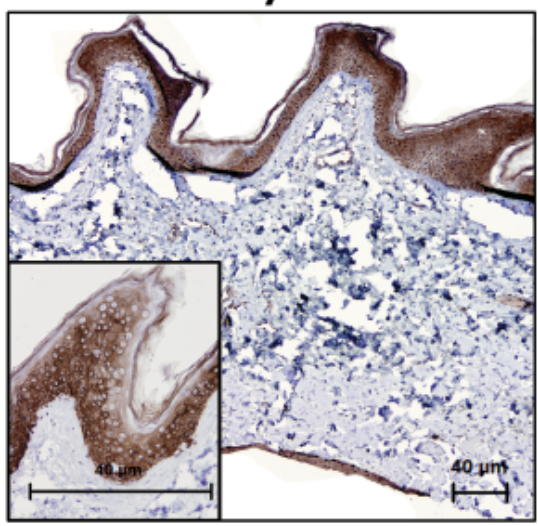

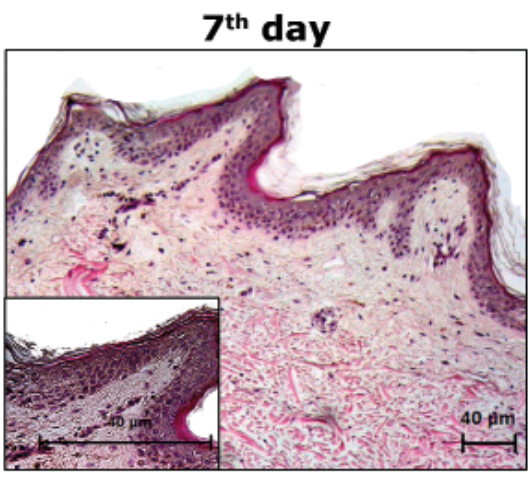

$7^{\text {th }}$ day

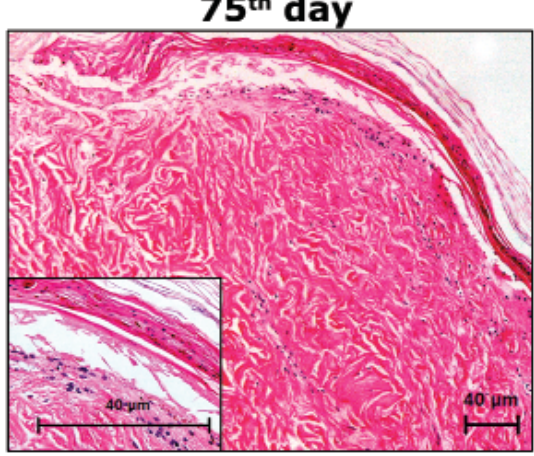

Ck10

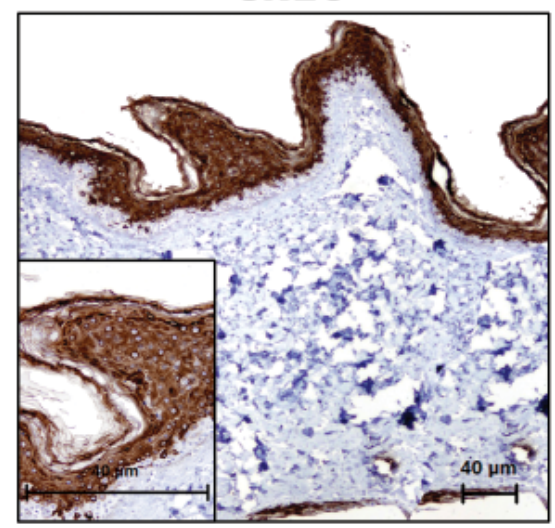

Figure 2: Hematoxylin eosin staining of skin on culture on days $1,7,30$ and 75 proving the viability of hOSEC method by the proliferative capacity of keratinocytes (magnification: 100x and 400x)

FIGURE 3: Immunohistochemical staining of skin on culture on day 75 for Ck5/6, Ck10 and Ki-67, proving the viability of hOSEC method by the proliferative capacity of keratinocytes (magnification: 100x and 400x) 


\section{DISCUSSION}

Animal testing for cosmetic products were banned in several countries and the industries in this field suffered serious constraints ${ }^{8}$. Therefore, research centers are attempting to develop alternative methods to animal use.

The hOSEC is a system of human skin in culture medium, which is the closest to in vivo condition because it maintains all skin structure such as melanocytes, Langerhans cells, keratinocytes, and the entire structure of dermal fibroblasts, glycosaminoglycans and collagen ${ }^{8}$. Due to these aspects it seems suitable to evaluate the safety and efficacy of topical products such as sunscreens for example, ${ }^{9}$ besides retaining important elements of in vivo skin, showing significant advantages for in vitro wound healing studies. ${ }^{10}$ It was initially described in studies on allergic contact dermatitis, lasting up to 3 weeks in culture. ${ }^{9}$

The present study demonstrated cell viability/vitality of human skin fragments in culture for 75 days. The hOSEC model's viability was confirmed by the maintenance of dermoepidermal junctions and epidermal proliferation, according to histological and immunohistochemical data for Ck5/6, Ck10 and the Ki-67 proliferation marker. Thus, this method is feasible for cosmetic testing up to 75 days. Moreover, its implementation is faster and less expensive than available in vitro systems.

However, this model has some limitations, such as the lack of innervation and circulation. Integration of blood vessels or the equivalent has been the subject of extensive research. ${ }^{11,12}$

\section{REFERENCE}

1. Report EC. Commission European Community Collaborative study on relationship between in vivo primary irritation and in vitro experimental models, 1988.

2. Draize J, Woodard G, Calvery H. Methods for the study of irritation and toxicity substances applied topically to the skin and mucous membrane. J Pharmacol Exp Ther. 1944;82:377-390.

3. Fentem JH, Briggs D, Chesné C, Elliott GR, Harbell JW, Heylings JR, et al. A prevalidation study on in vitro test for acute skin irritation: results and evaluation by the Management Team. Toxicol In Vitro. 2001;15:57-93.

4. Brosin A, Wolf $\mathrm{V}$, Mattheus A, Heise H. Use of XTT-assay to assess the cytotoxicity of different surfactants and metal salts in human keratinocytes (HaCaT). A feasible method for in vitro testing of skin irritants. Acta Derm Venereol. 1997;77:26-8.

5. Botham PA. Alternative methods for skin irritation testing: the current status. ECVAM Skin Irritation Task Force Report 1. ATLA. 1998;26:195-211.

6. Demetrulias J, Donnelly T, Morhenn V, Jessee B, Hainsworth S, Casterton P, et al. Skin2 - an in vitro human skin model: the correlation between in vivo and in vitro testing of surfactants. Exp Dermatol. 1998;7:18-26.

7. Jacobs JJ. Methyl green-pyronine staining of porcine organotypic skin explant cultures: an alternative model for screening skin irritants. ATLA. 2000;28:279-92.

8. Lebonvallet N, Jeanmaire C, Danoux L, Sibille P, Pauly G, Misery L. The evolution and use of skin explants: potential and limitations for dermatological research. Eur J Dermatol. 2010;20:671-84.
Communication in the skin is mediated by cytokines, neurotransmitters, hormones, growth factors and eicosanoids, which can be added to the culture medium although cell-to-cell interactions also appear to be necessary. ${ }^{8}$

The presence of all epidermal and dermal cellular types, immune cells such lymphocytes, dendritic cells, macrophages, and the conservation of the in vivo structure and physiology has permitted the use of skin explant in a large panel of diseases and processes, including immune diseases, wound healing, extracellular matrix process, ageing, cancer and cosmetic tests. Furthermore, it represents a good tool to understand physiopathology, and the lack of contact with the organism may be compensated by the addition of many substances. It remains the most representative in vitro model of human skin. ${ }^{8}$

\section{CONCLUSION}

The hOSEC model seems a good animal alternative method for safety assessment of cosmetic products for human uses. In addition, it can be regarded as being valid for a pre-clinical test similar to in vivo clinical human skin test because of the maintanance of its viability which was confirmed by immunohistological analysis. $\square$

\section{ACKNOWLEDGEMENTS}

Financial support: $\mathrm{CNPq}$ (Conselho Nacional de Desenvolvimento Científico e Tecnológico), FAPESP-PIPE phase 1 (Fundação de Amparo à Pesquisa do Estado de São Paulo - Programa de Incentivo à Pequena Empresa - fase 1) e CAPES (Coordenação de Aperfeiçoamento de Pessoal de Nível Superior)

9. Jacobs JJ, Lehé $\mathrm{CL}$, Hasegawa H, Elliott GR, Das PK. Skin irritants and contact sensitizers induce Langerhans cell migration and maturation at irritant concentration. Exp Dermatol. 2006;15:432-40.

10. Xu W, Jong Hong S, Jia S, Zhao Y, Galiano RD, Mustoe TA. Application of a partialthickness human ex vivo skin culture model in cutaneous wound healing study. Lab Invest. 2012;92:584-99.

11. Black AF, Berthod F, L'heureux N, Germain L, Auger FA. In vitro reconstruction of a human capillary-like network in a tissue engineered skin equivalent. FASEB J. 1998;12:1331-40.

12. Ponec M, El Ghalbzouri A, Dijkman R, Kempenaar J, van der Pluijm G, Koolwijk P. Endothelial network formed with human dermal microvascular endothelial cells in autologous multicellular skin substitutes. Angiogenesis. 2004;7:295-305.

M AILING ADDRESS:

A venida D ra. N adir A guiar, 1805

Jardim Paulo Gomes R omeo

14056-680 - Ribeirão Preto - SP

Brazil

E-mail: mandrey@fmrp.usp.br

How to cite this article: Frade MAC, Andrade TAM, Aguiar AFCL, Guedes FA, Leite MN, Passos WR, Coelho EB, Das PK. Prolonged viability of human organotypic skin explant in culture method (hOSEC). An Bras Dermatol. 2015;90(3):347-50. 